\title{
SUCCESSFUL MANAGEMENT OF PERIPARTUM CARDIOMYOPATHY IN A YOUNG FEMALE PATIENT
}

\author{
Ceren Yüksel ${ }^{1} \oplus$, Bengisu Çıray ${ }^{1} \oplus$, Mustafa Alperen Koşucu ${ }^{1} \oplus$, Servet Altay $^{2}$ ๑
}

${ }^{1}$ Trakya University School of Medicine, Edirne, TURKEY

${ }^{2}$ Department of Cardiology, Trakya University School of Medicine, Edirne, TURKEY

\begin{abstract}
Aims: Peripartum cardiomyopathy is a rarely seen pregnancy-related myocardial disorder. The diagnosis is usually challenging and is made by exclusion. We aimed to present the recovery of a patient with peripartum cardiomyopathy as a result of a successful treatment plan. Case Report: A 26-year-old female patient with type-2 diabetes mellitus presented to the cardiology department of Trakya University School of Medicine. The patient experienced dyspnea and edema after delivery at 37 weeks of gestation. After the results of the blood tests, further cardiac examinations were deemed necessary. The echocardiogram revealed a low ejection fraction, indicating heart failure. The patient was recommended to halt breastfeeding and bromocriptine treatment was started. The patient was discharged one week later and kept under follow-up. Conclusion: Peripartum cardiomyopathy is a rare disease and therefore not easy to diagnose, but with appropriate treatment plans and frequent follow-ups, patients have high chances of full recovery. Keywords: Peripartum, cardiomyopathy, pregnancy, bromocriptine, heart failure
\end{abstract}

\section{INTRODUCTION}

Peripartum cardiomyopathy (PPCM) is a type of heart failure that mostly affects previously healthy women in the puerperium, presenting with symptoms indicating congestion such as pulmonary rales, peripheral edema, and jugular vein distention (1). PPCM is characterized as left ventricular systolic dysfunction (LVSD) and ejection fraction (EF) below 45\%, which means that the blood pumped to the body is decreased and not sufficient (2). PPCM may heal on its own, but it can cause serious complications as it occurs with rapid clinical worsening. Garg et al. (3) state that although the underlying cause of the disease is not fully understood, multiparity, pregnancy over 30 years of age, race, twin pregnancy, obesity, hypertension, and eclampsia are among the risk factors. Apoptosis, the effect of prolactin hormone, endothelial mechanism disorders, autoimmune reactions, and inflammation are other reasons suggested for the development of this disease (4).

Peripartum cardiomyopathy is a rare clinical condition (1). As reported by Abboud et al. (5), according to individual studies that have been conducted, the incidence varies, with estimates ranging from 1 in 1,300 to 1 in 15,000. Additionally, Garg et al. (3) state that the prevalence is increased in people of African descent; the incidences estimated by studies are 1 in 1000 live births in South Africa, about 1 in 2289-4000 live births in the United States, and 1 in 299 live births in Haiti. Early diagnosis is difficult with LVSD and EF below $45 \%$, as symptoms are indeed common in almost all women after pregnancy (5). Although early diagnosis is difficult, it is a key factor in the prognosis of the disease, which could be life-saving for the patient (6).
We present the case of a patient with PPCM with a history of diabetes mellitus. As PPCM is a rare condition diagnosed by exclusion, we hope to contribute to the literature and raise awareness about PPCM.

\section{CASE REPORT}

A 26-year-old female patient with a history of type-2 diabetes mellitus presented to the cardiology department of Trakya University School of Medicine due to increased dyspnea and edema after delivery at 37 weeks of pregnancy. The patient was diagnosed with preeclampsia and edema at 34 weeks gestation. The patient was admitted to the cardiology service due to increased dyspnea. According to the patient's hemogram, her hemoglobin was $10.9 \mathrm{~g} / \mathrm{dl}$, her hematocrit value was 33.2\%, and her NT-ProBNP was $4753 \mathrm{pg} /$ $\mathrm{ml}$, which is significantly higher than the normal range (0-125 pg/ $\mathrm{ml}$ ), indicating heart failure. In the echocardiogram, the patient had an EF of 34\%. Global left ventricular hypokinesia, LVSD, left ventricular diastolic dysfunction, and left ventricle dilatation were observed. In addition to these findings, mild aortic insufficiency, mild tricuspid regurgitation, and moderate mitral regurgitation were detected. Because of the patient's history of diabetes, a selective coronary angiography was performed, and the angiogram showed no abnormalities. An endocrinology consultation was requested for the patient's treatment plan. After the consultation, bromocriptine treatment was started and breastfeeding was halted. In addition to bromocriptine, which is a dopamine receptor agonist, treatment for heart failure was started. Beta-blockers, diuretics, and angiotensin-converting enzyme inhibitors were used for the treatment 
of heart failure. After the consultation, endocrinology, nephrology, and cardiology follow-ups were recommended and the patient was discharged. She was prescribed $6.25 \mathrm{mg}$ of carvedilol, $25 \mathrm{mg}$ of spironolactone, $5 \mathrm{mg}$ of ramipril, $40 \mathrm{mg}$ of pantoprazole, $5 \mathrm{mg}$ of ivabradine, and insulin glulisine and insulin glargine.

Echocardiography and cardiac magnetic resonance imaging (MRI) were performed one month after discharge, and the patient had an EF of $45 \%$. Cardiac MRI showed depressed left ventricle contraction (Figure 1), and it was decided to continue the treatment. In the third month of the treatment, the patient became pregnant unintentionally, and a medical abortion was performed since she had an EF of $45 \%$. The patient was followed up in the sixth month and first year of her treatment. She had no complaints at her last follow-up and had an EF of $60 \%$.

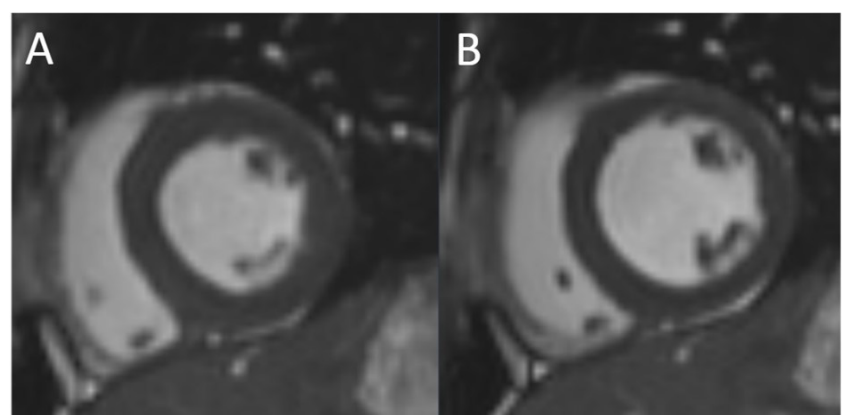

Figure 1: Cardiac MRI images in short axis steady-state free precession, depressed left ventricle contraction is observed. A: During systole. B: During diastole.

\section{DISCUSSION}

Diagnosing peripartum cardiomyopathy is difficult because its initial symptoms are common among pregnant and postpartum women. Moreover, PPCM presents without any previous heart-associated conditions and shows a rapid clinical worsening if not diagnosed on time and treated immediately. It is crucial to consult a cardiologist when a pregnant or postpartum patient applies to the hospital complaining of shortness of breath. After running diagnostic tests such as an echocardiogram, blood tests, and cardiac MRI, it should be evaluated whether the patient has PPCM, and a treatment plan should be established accordingly.

Besides the standard treatment for reduced EF, PPCM patients are also treated with a prolactin release inhibitor, bromocriptine (7). Bromocriptine usage on patients is rationalized by a hypothesis that claims that the heart of a postpartum woman would have increased oxidative stress levels. This oxidative stress would trigger the cathepsin D-mediated cleavage of prolactin into a $16-\mathrm{kDa}$ subform, thus affecting angiostatic and proapoptotic properties, benefitting myocardial microvascular injury, and blocking endothelial cell proliferation and migration (7). Considering that one of the primary goals of using bromocriptine as a therapy option of PPCM is to suppress prolactin release, discontinuation of breastfeeding is significant since breastfeeding stimulates prolactin.

European Society of Cardiology proposes a treatment plan called "the BOARD scheme" which implies that the treatment of PPCM should include bromocriptine, oral heart failure therapies, anticoagulants, vasorelaxant agents, and diuretics (8). In our case, the patient's medication regimen included beta-blockers (carvedilol), diuretics (spironolactone), angiotensin-converting enzyme inhibitors (ramipril), and $2.5 \mathrm{mg}$ of bromocriptine. Pantoprazole was prescribed for lowering the amount of acid released in the stomach. In order to slow her heart rate, the patient was prescribed ivabradine. Because our patient had type-2 diabetes mellitus, she continued using insulin glulisine and insulin glargine.

A review of case studies on the use of bromocriptine in peripartum cardiomyopathy patients conducted by Simon et al. (9) displays the recovery in low left ventricular EF when 2.5-5 mg of bromocriptine is used alongside standard heart failure therapy. Our patient's recovery process is also evidentiary to Simon et al. (9)'s review since her LVEF was first measured 30\%, and after using 2.5 $\mathrm{mg}$ bromocriptine daily, the LVEF was measured as $60 \%$ at the 6 thmonth follow-up.

Peripartum cardiomyopathy treatment is highly effective as the patients have a $50 \%$ chance of recovery and $98 \%$ chance of survival (9). Mortality rates differ regionally. As reported from single-center studies from Brazil and Haiti, their mortality rates are reported to be $14-16 \%$ in a month's interval (3). Biteker (10) stated that in two different studies investigating PPCM in Turkey, the mortality rate differs from $25 \%$ to $30 \%$.

Unfortunately, the recurrence rate of PPCM is high and patients are advised to seek counseling from a perinatologist in order to avoid future pregnancies (11). In our case, the patient got accidentally pregnant in the third month of the treatment process. Since the patient had an EF of approximately $45 \%$, which is not sufficient for keeping the patient's condition stable, a medical abortion had to be performed and their pregnancy was terminated. In conclusion, PPCM can cause heart failure, and even death, if not diagnosed on time and treated immediately. Therefore, it is cruicial not to overlook the possibility of peripartum cardiomyopathy, especially in young patients presenting with dyspnea or edema.

Ethics Committee Approval: N/A

Informed Consent: Informed verbal consent was obtained from the patient for this study.

Conflict of Interest: The authors declare no conflict of interest.

Author Contributions: Concept: CY, BÇ, MAK, SA. Design: CY, BÇ, MAK, SA. Supervision: CY, BÇ, MAK, SA. Resources: CY, BÇ, MAK, SA. Materials: CY, BÇ, MAK, SA. Data collection and/or Processing: CY, BÇ, MAK, SA. Analysis and/or Interpretation: CY, BÇ, MAK, SA. Literature Search: CY, BÇ, MAK, SA. Writing Manuscript: CY, BÇ, MAK, SA. Critical Review: CY, BÇ, MAK, SA.

Financial Disclosure: The authors declared that this study received no financial support.

Editor-in-Chief's Note: One of the authors of this article, Ceren Yüksel is a member of the editorial board of Turkish Medical Student Journal. However, she did not take place at any stage of the editorial decision of the manuscript. The editors who evaluated this manuscript are from other institutions.

\section{REFERENCES}

1. Koenig T, Hilfiker-Kleiner D, Bauersachs J. Peripartum cardiomyopathy. Herz 2018;43(5):431-7

2. Sliwa K, Hilfiker-Kleiner D, Petrie MC et al. Current state of knowledge on aetiology, diagnosis, management, and therapy of peripartum cardiomyopathy: a position statement from the Heart Failure Association of the European Society of Cardiology Working Group on peripartum cardiomyopathy. Eur J Heart Fail 2010;12(8):767-78.

3. Garg J, Palaniswamy C, Lanier GM. Peripartum cardiomyopathy: definition, incidence, etiopathogenesis, diagnosis, and management. Cardiol Rev 2015;23(2):6978.

4. Yamac H, Bultmann I, Sliwa K et al. Prolactin: a new therapeutic target in peripartum cardiomyopathy. Heart 2010;96(17):1352-7.

5. Abboud J, Murad Y, Chen-Scarabelli C et al. Peripartum cardiomyopathy: a comprehensive review. Int J Cardiol 2007;118(3):295-303.

6. Baris L, Cornette J, Johnson MR et al. Peripartum cardiomyopathy: disease or syndrome? Heart 2019;105(5):357-62.

7. Ballo P, Betti I, Mangialavori G et al. Peripartum cardiomyopathy presenting with predominant left ventricular diastolic dysfunction: efficacy of bromocriptine. Case Rep Med 2012 Nov 22. doi: 10.1155/2012/476903. [Epub ahead of print] 
8. Arrigo M, Blet A, Mebazaa A. Bromocriptine for the treatment of peripartum cardiomyopathy: welcome on BOARD. Eur Heart J 2017;38(35):2680-2.

9. Simon R, Yang S, Hameed AB. Bromocriptine use in peripartum cardiomyopathy: review of cases. AJP Rep 2018;8(4):e335-42.

10. Biteker M. Peripartum cardiomyopathy in Turkey. Int J Cardiol 2012;158(3):e60-1.

11. Elkayam U, Tummala PP, Rao K et al. Maternal and fetal outcomes of subsequent pregnancies in women with peripartum cardiomyopathy. N Engl J Med 2001;344(21):1567-71. 